\title{
Carnets
}

Revue électronique d'études françaises de l'APEF

Deuxième série - 3 | 2015

Insulaire

\section{L'île : un cadre propice au choc culturel}

Marcos Sarmiento Pérez et José Juan Batista Rodríguez

\section{(2) OpenEdition}

\section{Journals}

Édition électronique

URL : http://journals.openedition.org/carnets/1481

DOI : $10.4000 /$ carnets. 1481

ISSN : 1646-7698

Éditeur

APEF

\section{Référence électronique}

Marcos Sarmiento Pérez et José Juan Batista Rodríguez, «Lîle : un cadre propice au choc culturel », Carnets [En ligne], Deuxième série - 3| 2015, mis en ligne le 28 février 2015, consulté le 05 mai 2019. URL : http://journals.openedition.org/carnets/1481; DOI : 10.4000/carnets.1481

Ce document a été généré automatiquement le 5 mai 2019.

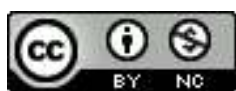

Carnets est mis à disposition selon les termes de la licence Creative Commons - Atribution - Pas d'utilisation commerciale 4.0 International. 


\title{
L'île : un cadre propice au choc culturel
}

\author{
Marcos Sarmiento Pérez et José Juan Batista Rodríguez
}

\section{NOTE DE L'AUTEUR}

Ce travail s'inscrit dans le cadre du projet de recherche FFI2011-25994 qui, sous le titre «El viaje a Canarias y sus escrituras II: catálogo digital de autores y textos », est financé par le Ministerio español de Economía y Competitividad (Plan nacional de I+D+I).

1 L'intérêt scientifique que suscitait pour la science l'état vierge des îles atlantiques jusqu'au milieu du $\mathrm{xx}^{\mathrm{e}}$ siècle, ou le simple attrait aventurier que les terres lointaines éveillaient chez l'Européen a donné lieu à une coïncidence où le «retard dans l'évolution » a côtoyé la plus grande « avancée » du monde occidental. C'est ainsi que les Insulaires et les Européens ont forgé leurs visions réciproques en se dévisageant mutuellement. C'est dans ce contexte, et en prenant comme référence l'île de Lanzarote aux Canaries, dans la seconde moitié du $\mathrm{XIX}^{\mathrm{e}}$ siècle, que nous analyserons les manifestations de l'affrontement culturel entre les Insulaires « ingénus » et « arriérés » et les représentants de cette première avancée scientifique européenne du moment, dans laquelle figurait le jeune zoologue suisse Hermann Fol qui effectua un séjour de trois mois à Lanzarote entre fin 1866 et début 1867.

2 Nous verrons que Fol fut par la suite l'un des zoologues les plus réputés de son pays ${ }^{1}$. Il avait choisi l'université d'Iena pour suivre ses études universitaires, malgré sa condition de francophone. À l'époque, Iena représentait, en Allemagne, l'avant-garde scientifique et encourageait la recherche dans le cadre de l'Évolutionnisme. En effet, depuis L'Origine des espèces, l'innovante œuvre de Charles Darwin, qui avait vu le jour à peine six ans auparavant, en 1859, et avait révolutionné la perspective de la science en écartant le point de vue créationniste considéré comme obsolète, le zoologue allemand Ernst Haeckel, professeur à Iena - où le jeune Hermann sera son élève - était devenu la figure la plus représentative du darwinisme sur le continent. Haeckel non content de défendre et 
diffuser l'œuvre de Darwin faisait un pas de plus en avant et dépassait le scientifique anglais : il se proposait de trouver le protorganisme ou l'organisme originel de tous les êtres vivants. C'est dans cet esprit qu'il arriva à Lanzarote en 1866, accompagné par ailleurs de Hermann Fol, de son collègue Richard Greef et d'un autre étudiant, le Russe Nikolai Nikoláievich Mikloukho-Maclay - qui deviendra plus tard l'un des scientifiques de renom de son pays grâce à ses recherches ethnographiques sur les Papous de NouvelleGuinée.

Quant à Hermann Fol, lorsqu'il arriva à Lanzarote à l'automne 1866, il trouva l'île atlantique à l'extrême opposé du niveau scientifique et culturel des pays de l'Europe centrale. Si l'Espagne était déjà plongée dans un retard séculaire aggravé par le déclin agonisant de sa politique coloniale, les îles Canaries, elles, de par leur éloignement du continent et leur manque de ressources, accusaient un retard encore plus grand. Et parmi les sept îles, c'est celle de Lanzarote qui était la plus sous-développée : manque d'eau, paysage aride, population peu nombreuse et isolée du monde extérieur. Les relations avec l'Europe étaient rares, car il n'existait pas de liaisons régulières par bateau. Il faut dire qu'une grande partie des Insulaires ne connaissaient même pas Fuerteventura, l'île canarienne voisine alors que celle-ci n'est séparée de Lanzarote que par un bras de mer. Seules de petites embarcations mouillaient dans le port d'Arrecife, la «capitale » de Lanzarote. C'était, en majorité, des bateaux espagnols et, durant les trois mois que Fol et ses accompagnateurs ont séjourné dans l'île, c'est à peine si deux ou trois navires anglais sont passés par là, en route vers la côte africaine. En un mot, la petite île manquait pratiquement de tout ce qui pouvait être en rapport avec l'idée de progrès.

4 Cette sensation de régression, Fol et ses accompagnateurs la ressentirent dès qu'ils débarquèrent aux Canaries: après avoir laissé derrière eux le confort européen, ils avaient pu profiter de deux journées magnifiques à Madère qui, à l'époque, possédait une merveilleuse infrastructure touristique bien connue de la classe aisée européenne. Ils avaient visité Funchal et firent une excursion à Câmara de Lobos, une ville voisine (Greeff, 1868: 111-112). À Ténériffe, où ils ne trouvèrent pas l'opulence de l'île portugaise, ils eurent néanmoins la belle occasion de monter jusqu'au Teide. Une ascension que le jeune genevois ne put mener jusqu'au bout suite à la ruade au genou de la part d'une mule alors qu'il se trouvait à mi-chemin du sommet (Greeff, 1868:196). L'accueil qu'on leur réserva fut aimable. Ils reçurent par ailleurs les sages conseils d'un autre Suisse résidant dans l'île, Hermann Wildpret, et surtout ceux de Sabin Berthelot ${ }^{2}$, à l'époque consul de France dans l'île (Haeckel, $1867: 315$ ). Finalement, après quatre jours de navigation difficile sur un petit voilier qui progressait lentement, ils débarquèrent sur l'île déserte de Lanzarote, et les attentes des quatre scientifiques européens se réduisirent encore plus substantiellement. Le terrain était bel et bien amendé pour le choc culturel.

5 En effet, nos insignes Européens d'Europe centrale ont dû faire face dans l'humble Arrecife à une population d'environ 3.000 habitants $^{3}$ vivant de la production et de la préparation de la cochenille et en moindre mesure de la barrilla (Chénopodiacées) (Haeckel, $1923: 60)^{4}$. Comme le reste de l'île, la ville manquait beaucoup d'eau et il fallait

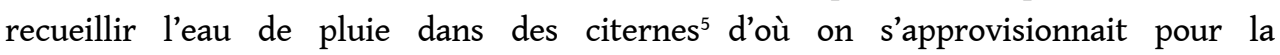
consommation journalière. Il n'était d'ailleurs pas rare, lorsque les périodes de sécheresse se prolongeaient, de faire venir de l'eau en bateau de la Grande Canarie ou de Ténériffe (Fritsch, 2006 : 176-177).

6 Après s'être hébergés les premiers jours dans deux pensions de l'île, les problèmes surgirent lorsqu'ils s'avisèrent de trouver un logement plus approprié qui devait aussi 
leur servir de laboratoire pour leurs recherches. C'est avec beaucoup de difficultés qu'ils parvinrent à louer une maison dans la rue Principal et ils durent venir à bout d'énormes inconvénients pour meubler et équiper le laboratoire. Ils eurent donc recours à un menuisier local pour la confection d'une grande table afin de subvenir aux besoins et leur permettre à la fois de lire, écrire, analyser au microscope, dessiner, disséquer et étudier les états évolutifs des animaux marins, etc. Tout aussi difficile fut l'acquisition des ustensiles indispensables pour le travail de zoologie - à savoir, trouver des récipients en verre -, car ils n'en avaient pas apporté en nombre suffisant, pensant qu'ils en trouveraient sur place ${ }^{6}$. Par ailleurs, comme ils se trouvaient dans l'impossibilité de compter sur un service domestique, ils durent se charger eux-mêmes des tâches habituelles comme puiser de l'eau dans la citerne, la distiller dans le petit bassin en pierre poreuse, laver leur linge, faire leur lit, etc. Le comble, c'est qu'il n'y avait guère de livres dans la ville et qu'on pouvait y trouver uniquement trois journaux : el Diario español et deux gazettes, l'une imprimée à Ténériffe et l'autre à la Grande Canarie (Haeckel, 1923 : $60)$.

7 On ne s'étonnera pas que cet isolement par rapport à la culture européenne ait provoqué chez nos scientifiques la sensation de se retrouver dans une île lointaine d'Océanie (Haeckel, 1923: 57) et que cela fit surgir des différences culturelles ainsi que des préjudices auxquels le retard culturel des Insulaires avait, sans nul doute, lui aussi contribué. Au début, durant les trois premiers mois que Fol et ses accompagnateurs séjournèrent à Arrecife - où de petits gorets ornés d'un ruban rouge autour du cou courraient aussi librement dans les rues de la ville que le font les chiens en Allemagne - , les quatre naturalistes fréquentèrent à peine la population du lieu. Leurs amis se réduisaient à ceux que nous avons cités, à savoir M. José Barón, $\mathrm{M}$. Topham, le vice-consul anglais ${ }^{7}$, Domingo, le propriétaire de la pension où ils prenaient leurs repas, et les deux propriétaires des canots utilisés pour effectuer leurs petites excursions maritimes, qui se prénommaient Florencio et Juan. Selon Haeckel, les habitants d'Arrecife étaient de bonnes gens; ils étaient inoffensifs, mais ignoraient les ombres et les lumières de la civilisation européenne. Leur niveau de formation globale était très bas (Haeckel, 1923 : 60), et même les personnes les plus distinguées et les plus cultivées parmi eux avaient une idée étrange de l'Europe et notamment de l'Allemagne.

Aux yeux des naturalistes, les habitants de Lanzarote étaient une espèce de grands enfants, avec tous les vices et les vertus des enfants européens âgés de 10 et 12 ans : ils se passionnaient pour les jeux de hasard, ils ignoraient toute occupation sérieuse et travail rigoureux, ce qui les amenait à passer pratiquement toute la journée dans l'oisiveté, à bavarder ou à jouer dans la rue ou devant leur porte, qui servait en même temps d'unique fenêtre. Quant aux femmes, elles restaient enfermées à la maison et ne sortaient généralement que le dimanche après-midi. À tout cela s'ajoutait un autre aspect bien typique des habitants d'Arrecife, leur manque de ponctualité à laquelle Haeckel fait allusion en parlant de "perte de temps avec laquelle il fallait compter à chaque fois que l'on demandait quelque chose » (Haeckel, $1923: 54)^{8}$.

9 Par ailleurs, du point de vue de cette perspective insulaire et étant donné qu'Arrecife présentait peu de possibilités de loisir, les quatre naturalistes allemands traitèrent le sujet de conversation le plus intéressant qui soit durant cet hiver passé dans l'île. On ne cessait d'ailleurs de fabuler et de se faire des idées constamment à leur sujet : l'opinion générale était que les recherches zoologiques des quatre Européens n'étaient qu'une mascarade et que le véritable objectif de leur séjour était d'épier l'île pour le compte du gouvernement 
prussien dont l'intention était de conquérir l'archipel avec sa flotte. Pour d'autres, il s'agissait de Français camouflés. Et en effet, même si dans le cas présent nous reprenons des informations provenant des naturalistes eux-mêmes, il n'était pas rare que les paysans canariens considèrent les étrangers comme des espions, c'est du moins les propos que le naturaliste français de Ténériffe Sabin Berthelot aura à ce sujet :

L'étranger (...) s'il dessine, c'est qu'il est en train de faire le lever d'un plan et qu'il se propose d'acquérir des terres; s'il prend des notes, on parle tout bas pendant qu'il écrit et on se tient à distance, car il est sûrement un personnage important, un agent secret d'une quelconque puissance qui a des vues sur l'île (2006 [1839]: 192-193).

Il s'agit là d'une image si enracinée dans les îles que Berthelot lui-même l'avait critiquée chez l'illustre Canarien Viera y Clavijo lequel fut le premier à considérer George Glas comme « un homme suspect pour le pays » (1997 [1839] : 61-62).

D’autre part, comme il était notoire que les quatre naturalistes n'étaient pas catholiques, cela signifiait automatiquement, pour les habitants de Lanzarote, qu'ils n'étaient pas chrétiens non plus. À cette idée contribuait le fait que leurs instruments de laboratoire leur avaient donné une renommée d'hommes attachés à la sorcellerie. On pensait d'ailleurs qu'ils étaient probablement protégés par un onguent magique contre toutes les maladies car ils jouissaient d'une santé à faire envie même si on leur avait auguré dès le début du séjour que leurs bains nocturnes quotidiens allaient indéfectiblement les tuer. Et vu qu'on les prenait pour des sorciers, plus d'un habitant se déplaça des différentes localités de l'île pour venir les consulter. En un mot, dès que les Allemands blancs Alemano blanco ${ }^{9}$ faisaient leur apparition dans la rue ou dans la pension, les habitants d'Arrecife les observaient et les saluaient avec un mélange profond de respect, d'admiration, de crainte et d'aversion. Et lorsque, le soir, ils se dirigeaient vers la plage, les enfants surpris et craintifs, fuyaient en courant vers leurs baraques.

Devant de telles considérations "superstitieuses" de la majorité des habitants, les propriétaires de canots, qui connaissaient bien les naturalistes puisqu'ils les servaient tous les jours, se sentaient très flattés de servir des personnes d'un si haut rang professionnel.

\section{Conclusions}

13 Il n'est pas étonnant qu'en dépit de leur reconnaissance pour les trésors zoologiques que la mer de Lanzarote leur avait fournis (Haeckel, 1923: 66-67), les quatre naturalistes abandonnèrent l'île avec un " plus jamais se revoir » et ce n'est pas étonnant non plus que Haeckel écrive en arrivant à Gibraltar :

Hier, dimanche 17 mars, à 03 h00 de l'après-midi (...) j'ai remis pied à terre sur le sol européen. Vous ne pouvez pas vous imaginer la sensation éblouissante et captivante que nous a causé Gibraltar, la première ville dotée de culture européenne (...), et après n'avoir vu pendant trois mois que les misérables et ennuyeuses baraques de Lanzarote, et en dernier lieu, les villes portuaires primitives de la côte Nord-Occidentale (...) Même le menu frugal de notre auberge (...) nous sembla un festin après avoir mangé pendant 15 semaines quasi exclusivement du poisson frit dans de l'huile rance, de la viande dure comme une semelle et, comme uniques plats savoureux, des fruits et des œufs. À présent, nous connaissons la situation des gens non civilisés en dehors de l'Europe (Haeckel, $1923: 79)$. 
14 pointait à l'égard des Insulaires : ils admettaient par exemple leur adresse extraordinaire (Haeckel, 1867: 317) et reconnaissaient aussi le splendide climat de l'île - ce qui compensait l'image du paysage désertique et inhospitalier impropre aux excursions -, la rareté de l'eau, l'état primitif des maisons, l'absence de stimulations culturelles ou encore le temps monotone des journées passées à Arrecife.

15 Il est fort probable que, dans un premier temps, la relation avec Sabin Berthelot à Ténériffe et plus tard avec José Barón à Arrecife, tous les deux représentants de la culture européenne aux Canaries, ait contribué à atténuer les effets du choc culturel.

16 Malheureusement, nous ne disposons pas de témoignages directs provenant d'habitants de Lanzarote, ce qui nous oblige à considérer les deux points de vue d'une même réalité uniquement en partant de la perspective de Haeckel et Greeff, car nous n'avons même pas pu trouver d'arguments directs chez Fol et Nikloukho-Maclay. Il faut dire que même dans les perceptions de Haeckel et Greeff on constate des différences appréciables. Le premier nous transmet directement les informations dans des lettres qu'il adressa de Lanzarote à ses parents, amis et collègues et qui furent publiées telles qu'elles avaient été écrites. Greeff, par contre, utilisa ses lettres en guise d'orientation pour ensuite les passer par le filtre d'une adaptation et nous laisser l'information sur son voyage dans un récit, évitant ainsi les nombreuses lacunes et l'excessive exhaustivité des lettres (Greeff, 1868: Vorwort).

\section{BIBLIOGRAPHIE}

ÁLVAREZ RIXO, José Agustín (1982). Historia del puerto del Arrecife en la isla de Lanzarote, una de las Canarias. Santa Cruz de Tenerife : Aula de Cultura del Cabildo Insular de Tenerife - Instituto de Estudios Canarios.

BATISTA RODRíGUEZ, José Juan (2007). « Sabin Berthelot : la mediación cultural francesa », in José Oliver Frade, Alberto Relancio Menéndez (eds. ). El descubrimiento científico de las Islas Canarias. Santa Cruz de Tenerife : Fundación Canaria Orotava de Historia de la Ciencia, pp. 143-159. BEDOT, Maurice (1894). Hermann Fol. Sa vie et ses travaux. Genève : Aubert-Schuchardt. BERTHELOT, Sabin (1997 [1839]). Misceláneas Canarias (traducción de Manuel Suárez Rosales y estudio crítico de Manuel Hernández González). La Laguna : Francisco Lemus Editor.

BERTHELOT, Sabin (2006 [1839]). Historia natural de las Islas Canarias (Geografía descriptiva, estadística y geología) (traducción de José A. Delgado Luis y estudio crítico de Manuel Hernández). La Orotava : J.A.D.L./Ayuntamiento de La Orotava.

BuSCAGliA, Marino, DUboule, Denis (2002). « Development Biology in Geneva: A Three CenturyLong Tradition », The International journal of developmental biology, $\mathrm{n}^{\circ} 46, \mathrm{pp}$. 5-13.

FOL, Hermann (1869). Ein Beitrag zur Anatomie und Entwickelungsgeschichte einiger Rippenquallen. Inaugural-Dissertation zur Erlangung der Doktorwürde in der Medicin und Chirurgie der medicinischen Fakultät der Friedrich-Wilhelms-Universität zu Berlin. Berlin : Brüxenstein. 
FRITSCH, Karl von (2006). Las Islas Canarias. Cuadros de viaje (traducción, estudio introductorio y Notas de José Juan Batista Rodríguez y Encarna Tabares Plasencia). Santa Cruz de Tenerife : Centro de la Cultura Popular Canaria.

GREEFF, Richard (1868). Reise nach den Canarischen Inseln. (London, Lissabon, Madeira, Gran Canaria, Lanzarote, Marokko, Spanien). Mit populär-naturwissenschaftlichen Schilderungen. Bonn : Max Cohen \& Sohn.

HAECKEL, Ernst (1867). « Eine zoologische Excursion nach den Canarischen Inseln. Vorläufiger Reisebericht ", Jenaische Zeitschrift für Medicin und Naturwissenschaft (herausgegeben von der medicinisch-naturwissenschaftlichen Gesellschaft zu Jena), Dritter Band, pp. 313-328.

HAECKEL, Ernst (1870). « Eine Besteigung des Pik von Teneriffa », Zeitschrift der Gesellschaft für Erdkunde zu Berlin, Fünfter Band, pp. 1-28.

HAECKEL, Ernst (1923). « Reise nach den Kanarischen Inseln (1866/67) », Berg- und Seefahrten 1857-1883, pp. 27-80.

Historisches Lexikon der Schweiz, Bd. 4 (2005). Basel : Schwabe Verlag.

NDB = Neue Deutsche Biographie (1875-1912). Leipzig : Duncker \& Humblot.

QUINTANA NAVARRO, Francisco (1992). Informes consulares británicos sobre Canarias (1856-1914). 2 vol. Las Palmas de Gran Canaria : La Caja de Canarias.

SARMIENTO PÉREZ, Marcos (2011). La expedición científica de Ernst Haeckel a Lanzarote (1866-67). Las

Canarias en la teoría de la evolución. Málaga : Libros ENCASA.

\section{NOTES}

1. Cependant, nous exposerons tout d'abord quelques aspects de la biographie, brève mais brillante, de notre protagoniste qui souligneront le contraste avec l'expérience vécue à Lanzarote. Fol était né en 1845 à Saint-Mandé, près de Paris. Ses parents étaient de Genève, ville où il fit ses études secondaires et ses deux premières années universitaires en zoologie (Buscaglia \& Duboule, 2002 : 9). Attiré par la popularité de Haeckel, en 1864, il poursuivit ses études à Iéna. C'est là que Haeckel le choisit, lui et Nikolai Mikloukho-Maclay, pour l'accompagner dans cette île atlantique solitaire. Il eut là son premier contact avec la mer et entreprit l'étude de la zoologie marine. Ses recherches sur le terrain allaient donner lieu plus tard à une thèse de doctorat sur l'anatomie et le développement des cténophores (Fol, 1869: 13), ce qui l'amena à approfondir l'embryologie descriptive des invertébrés (Buscaglia \& Duboule, $2002: 10$ ).

De retour à Genève en 1870, Fol installa un laboratoire à Messine, et créa plus tard la station zoologique de Villefranche-sur-Mer où il allait passer les années les plus productives de sa carrière scientifique. C'est de cette époque que datent ses "Études sur le développement des Mollusques » qui mirent en avant sa maîtrise de la technique microscopique acquise dans le laboratoire rudimentaire de Lanzarote.

$\mathrm{Au}$ fur et à mesure que Fol avançait dans l'embryologie, il se sentait attiré par les problèmes de fécondation. En 1878, il publiait déjà Recherches sur la fécondation et le commencement de l'Hénogénie chez divers animaux, une œuvre dans laquelle il défendait la théorie cellulaire de l'ovule et contestait la théorie de la vésicule germinative en tant que véritable cellule. Il fut, dans ce contexte, le premier à observer au microscope la fécondation d'un ovule d'étoile de mer en 1877 (Historisches Lexikon der Schweiz, 2005 : 593).

En 1878 il occupa la chaire d'embryologie comparée et de tératologie de l'Université de Genève à laquelle il dédia un ouvrage intitulé Genève et son Université -, et dirigea aussi Recueil 
Zoologique Suisse qui contribua au renom scientifique de son pays. C'est lui aussi qui fonda la Revue Suisse de Zoologie et Annales du Musée d'Histoire Naturelle de Genève, dont le premier numéro parut en 1893, un an après son décès.

En 1886, revivant sans doute sa première expérience dans les eaux de Lanzarote, il abandonna la chaire de l'université et revint s'établir à Villefranche-sur-Mer où le gouvernement français le nomma directeur-adjoint de la Station zoologique (Bedot, 1894: 8). À bord du petit vapeur l'Amphiaster, il se consacra à ses recherches préférées au large des côtes de Nice et de la Corse. Toutefois, l'étude de la fécondation resta l'une de ses principales préoccupations. C'est ainsi que dans l'un de ses derniers travaux importants, «La quadrille des centres, un épisode nouveau dans l'histoire de la fécondation ", il décrit les transformations qui se produisent dans le noyau spermatique et l'ovocyte.

Dans le but de trouver de nouvelles applications pour la description d'objets observés au microscope, il effectua plusieurs études sur la photographie qu'il publia dans la Revue suisse de photographie et dans Nature. Fol fut cofondateur de la Société photographique de Genève et fut membre de plusieurs sociétés scientifiques: La Société impériale des amis de la Science de Moscou, La Société belge de microscopie, la Société royale de microscopie de Londres, la Société néerlandaise de zoologie et l'Académie Léopoldine des naturalistes allemands. Le Gouvernement français lui décerna la Grande Croix de la Légion d'Honneur pour avoir créé la station zoologique de Villefranche-sur-Mer (Bedot, 1894 : 8). Considéré comme le père de la cytologie moderne, Fol a publié plus de 140 articles dont 49 sur l'embryologie et 15 sur la fertilisation.

En 1892, à 47 ans, sa vie s'achève prématurément et tragiquement en mer - cette mer qui avait été son milieu comme zoologue dans l'île canarienne. Cette année-là, le Ministre français de l'Instruction publique lui avait commandé une monographie sur les éponges en Méditerranée. Il appareilla du port du Havre et arriva quelques jours plus tard dans la petite localité bretonne de Bénodet. C'est à partir de là que l'on perd sa trace. Vingt ans auparavant, en 1873, il avait collaboré avec son père et son frère Walter à la fondation du Musée Fol de Genève qui devint en 1910 le Musée d'art et d'histoire.

2. Marseillais de naissance, Berthelot partagea les quatre-vingt-six ans de sa vie entre son pays et Ténériffe, l'île où il reçut le titre de «fils adoptif » et où il mourut. Co-auteur de la monumentale Histoire naturelle des Îles Canaries (1836-1850), un ouvrage qui a servi de véritable lien entre les Canaries et l'Europe, Berthelot a favorisé le contact entre de nombreux étrangers et la réalité de l'archipel des Canaries (cf. Batista Rodríguez, 2007 : 143-159).

3. Karl von Fritsch (2006: 162-163) note 2.699 habitants, mais Álvarez Rixo (1982: 215), qui renvoie à l'information donnée par le journal La Aurora, publié à Santa Cruz en 1848, parle déjà de plus de 3.000 âmes pour cette année-là.

4. La cochenille avait été introduite dans l'île vers 1831 ; en 1839 on l'exportait déjà du port d'Arrecife (Álvarez Rixo, 1982 : 207).

5. Au début de l'année, à l'arrivée de nos voyageurs dans cette localité, on note un fait curieux : il avait plu en abondance. «Le 6 janvier 1866, la pluie ne cessa de tomber de 8 heures du soir jusqu'à l'aube du lendemain. Il plut avec une telle force dans les environs du port d'Arrecife que les citernes débordèrent et que l'eau coula vers la ville et inonda une distillerie de M. André Lemes et lui causa des dommages » (Álvarez Rixo, 1982 : 228).

6. Fort heureusement, pour trouver une maison - que les habitants d'Arrecife appelèrent «la maison des quatre naturalistes ", et qui se trouvait à deux pas de la plage - de même que pour acquérir les ustensiles indispensables, l'aide de M. José Barón, un commerçant local d'ascendance italienne qui parlait le français en plus de l'espagnol et l'italien, s'avéra providentielle (Haeckel, 1923 : 56-57).

7. Bien que Haeckel fasse allusion à John Thomas Topham en tant que "consul ", en réalité cet entrepreneur renommé était vice-consul à Arrecife depuis plus de six ans (Quintana Navarro, 1992 : I, XXXIX). Quant à ce patronyme anglais, on apprend par Álvarez Rixo (1982 : 113, 119 y 
198) qu'il y avait parmi les édiles d'Arrecife un nommé Guillermo Topham, qui fut maire en 1819 puis en 1828, en plus de sa fonction de procureur pour négocier des affaires, en 1824.

8. Ce commentaire vient de Haeckel à propos d'une des excursions qu'il effectua dans l'île, à Haría. En effet les chameaux devaient être prêts à sept heures du matin mais ils n'arrivèrent pas avant neuf heures.

9. Dans son texte, Haeckel écrit « weisse Deutsche », en allemand, c'est-à-dire, Allemands blancs, et, entre parenthèses, Alemano blanco.

\section{RÉSUMÉS}

Entre fin 1866 et début 1867, le jeune zoologiste suisse Hermann Fol - qui, plus tard, sera l'un des embryologistes les plus réputés de son pays - a mené des recherches dans l'île de Lanzarote, aux Canaries, pendant trois mois. Il accompagnait l'un de ses professeurs de l'Université de Iéna (Allemagne), Ernst Haeckel, à l'époque considéré comme le meilleur représentant scientifique du récent darwinisme sur le continent européen. Dans le cadre de l'intérêt scientifique que suscitaient les terres vierges des îles atlantiques chez les scientifiques, nous analysons l'écart culturel entre les natifs "candides» et "arriérés» et les représentants d'une avancée scientifique européenne de l'époque. Nous complétons ce travail avec une ébauche biographique du zoologue genevois, ainsi que les résultats de sa recherche dans l'île qui ont donné lieu à sa thèse de doctorat sur l'anatomie et le développement des cténophores, ce qui a constitué ses débuts dans l'embryologie descriptive des invertébrés.

From late 1866 to early 1867, young Swiss zoologist Hermann Fol, who later became one of Switzerland's most eminent embryologists, spent three months in Lanzarote, in the Canary Islands. He was accompanying Ernst Haeckel, one of his lecturers from the University of Jena (Germany) and leading supporter in continental Europe of the recently emerged Darwinism. In the context of the scientific interest that the natural, untouched condition of the Atlantic islands aroused among scientists, we examine the clash of cultures between the "naïve" and "slow" islanders and those at the forefront of European science at the time. The study is completed by a biographical outline of Fol and the results of his research in the island, which led to his doctoral thesis on the anatomy and development of the ctenophore and consolidated his entry into descriptive embryology of invertebrates.

\section{INDEX}

Mots-clés : Lanzarote, choc culturel, Fol (Hermann), îles atlantiques, darwinisme

Keywords : Lanzarote, clash of cultures, Fol (Hermann), Atlantic islands, Darwinism

\section{AUTEURS}

\section{MARCOS SARMIENTO PÉREZ}

Université de Las Palmas de Gran Canaria

msarmiento[at]dfm.ulpgc.es 
JOSÉ JUAN BATISTA RODRÍGUEZ

Université de La Laguna

jjbatist[at]ull.es 